\title{
Nanotechnological Approach to Increase the Antioxidant and Cytotoxic Efficacy of Crocin and Crocetin
}

\author{
Authors \\ Carmelo Puglia ${ }^{1}$, Debora Santonocito ${ }^{1}$, Teresa Musumeci ${ }^{1}$, Venera Cardile ${ }^{2}$, Adriana Carol Eleonora Graziano ${ }^{2}$, \\ Loredana Salerno ${ }^{1}$, Giuseppina Raciti ${ }^{1}$, Lucia Crascì ${ }^{1}$, Anna Maria Panico ${ }^{1}$, Giovanni Puglisi ${ }^{1}$
}

Affiliations

1 Department of Drug Sciences, University of Catania, Catania, Italy

2 Department of Biomedical and Biotechnological Sciences, University of Catania, Catania, Italy

Key words

Crocus sativus, Iridaceae, solid lipid nanoparticles, Turbiscan analysis, antioxidant, cytotoxic

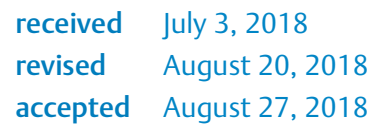

\section{ABSTRACT}

Crocin and crocetin are two interesting constituents of saffron (Crocus sativus) that possess important biological activities. Their use as therapeutic agents is strongly compromised by a scarce stability, poor absorption, and low bioavailability. Therefore, to improve these unfavorable features, the aim of the present work has been to apply a nanotechnological approach based on the formulation of solid lipid nanoparticles containing crocin and crocetin. Solid lipid nanoparticles were formulated according to crocin and crocetin chemical properties, using a variation of the quasi-emulsion solvent diffusion method to formulate crocin-solid lipid nanoparticles, while crocetin-solid lipid nanoparticles were prepared following the solvent diffusion method. Morphology and dimensional distribution of solid lipid nanoparticles have been characterized by differential scanning calorimetry and photon correlation spectroscopy, respectively, while the effect of drug incorporation versus time has been studied by Turbiscan technology. In order to verify the role of the nanotechnological approach on the biological activities of crocin and crocetin, the antioxidant and antiproliferative effects of these carotenoids once incorporated in lipid nanoparticles have been evaluated. For this aim, the oxygen radical absorbance capacity assay and the MTT test were used, respectively.

The results pointed out the formulation of nanometric dispersions endowed with high homogeneity and stability, with an encapsulation efficiency ranging from 80 (crocetin-solid lipid nanoparticles) to $94 \%$ (crocin-crocetin). The oxygen radical absorbance capacity assay evidenced an interesting and prolonged antioxidant activity of crocin and crocetin once encapsulated in solid lipid nanoparticles, while the nanoencapsulation strategy showed a different mechanism in ameliorating the cytotoxic effect of these two substances.

\section{Introduction}

Saffron, dry stigmas of Crocus sativus L. (Iridaceae) flowers, has been studied for many therapeutic applications including antioxidant and cytotoxic properties [1,2]. This spice contains bioactive constituents such as picrocrocin, CRO, and CRT, which belong to the family of carotenoids [3].

Carotenoids are a group of lipophilic pigmented natural substances formed by carotenes and their derivatives. The basic structure of carotenoids is made up of eight isoprenoids combined with a series of double-bonded conjugates. Carotenoids can have vitaminic activity, and can perform an immunomodula- tory function in relation to the strong chain-breaking antioxidants capacity. They act as free radical scavengers by donating hydrogen or transferring a single electron to radicals. Through this action, carotenoids can strengthen the immune system and also block the progress of precancerous diseases.

CRO and CRT ( Fig. 1), in particular, possess interesting biological activities. CRT is an amphiphilic low molecular weight carotenoid showing excellent antioxidant, anti-atherosclerotic, antiinflammatory, and anticancer activities in vitro and in vivo [4,5]. $\mathrm{CRO}$, the water-soluble derivative of CRT, has high antioxidant activity [6-8] and shows beneficial effects on many organs such as 


$\begin{array}{ll}\text { ABBREVIATIONS } \\ \text { AAPH } & \text { 2,2'-azobis(2-amidinopropane) dihydrochloride } \\ \text { CRO } & \text { crocin } \\ \text { CRT } & \text { crocetin } \\ \text { DR } & \text { drug recovery } \\ \text { DSC } & \text { differential scanning calorimetry } \\ \text { FL } & \text { fluorescein } \\ \text { HPMC } & \text { (hydroxypropyl) methyl cellulose } \\ \text { ORAC } & \text { oxygen radical absorbance capacity } \\ \text { PCS } & \text { photon correlation spectroscopy } \\ \text { PDI } & \text { polydispersivity index } \\ \text { QESD } & \text { quasi-emulsion solvent diffusion } \\ \text { SLNs } & \text { solid lipid nanoparticles } \\ \text { TSI } & \text { Turbiscan stability index }\end{array}$

the nervous, cardiovascular, and immune systems and against cancer [9-13].

In spite of such beneficial activities of both CRT and CRO, their use is limited because of their unfavorable physical and chemical features. CRO, for instance, is scarcely stable. Indeed, it loses most of its functionality after exposure to heat, oxygen, light, and acids $[14,15]$. It shows a poor absorption and low bioavailability after oral administration, being hydrolyzed by $\beta$-glucosidase and rapidly eliminated [16]. On the contrary, the biological application of CRT is mainly limited due to its hydrophobic nature and poor aqueous solubility [17].

Nanoscale drug delivery systems are an important tool to overcome the aforementioned limitations and to improve the pharmacokinetics and bioavailability of drugs and natural active compounds [18].

SLNs, which are members of the lipid nanocarriers family, are able to solubilize hydrophilic and lipophilic molecules in a physiologic environment, controlling their delivery and protecting them from degradation [19]. Compared with other systems, SLNs have many benefits, such as ease of preparation and low cost of raw materials, high-scale production, excellent physical stability, and biodegradability of lipids [20]. These favorable properties of SLNs make them promising nanocarriers to overcome or at least minimize some of the drawbacks of conventional formulations.

A general recognized limit of SLNs is represented by the entrapment of a hydrophilic drug into the lipid matrix of the nanoparticle. This condition is often characterized by a low payload and a scarce bioacceptability due to the use of organic solvents during the formulation [21].

The aim of this study was the preparation and characterization of CRO and CRT containing SLNs, and the evaluation of the antioxidant and antiproliferative effects of these carotenoids once incorporated into lipid nanoparticles. For this aim, the ORAC assay and the MTT test were used, respectively.

CRO-loaded SLNs, in spite of the hydrophilic character of CRO, were formulated by using a modification of the QESD technique, a particular approach to the general solvent-change method [22].

\section{Results and Discussion}

The methods used to formulate CRO- and CRT-loaded SLNs have proven to be valid and highly reproducible despite the instability of the molecules. CRO-SLNs and CRT-SLNs were prepared with Softisan 100 (hydrogenated coco-glycerides) and Pluronic F68 (poloxamer 188) as solid lipid and surfactant, respectively. We decided to use hydrogenated coco-glycerides after a screening to identify the best lipid matrices for CRO and CRT incorporation. These lipids are characterized by a low melting point $\left(35^{\circ} \mathrm{C}\right)$, a very important feature to prepare the SLNs, thus avoiding thermal stress conditions. The formulation strategies we used gave interesting results as confirmed by PCS analysis and drug loading determination ( $\vee$ Table 1 ). SLNs prepared by the multiple emulsion technique showed a mean diameter of $270 \mathrm{~nm}$, a PDI value around 0.15 , and a DR\% of 82.1 , while CRT-SLNs, formulated by the solvent diffusion technique, showed acceptable results regarding dimensional analyzes: the mean diameter was around $280 \mathrm{~nm}$ and the PDI value around 0.30 , while the DR was about $94 \%$ ( $\vee$ Table 1 ). As regards to zeta potential values, we obtained - 17.8 mV for CRT-SLNs and - 30.2 for the CRO-SLNs, predicting a good long-term stability for both formulations ( $\vee$ Table 1 ).

In addition, we assessed the physical stability of all the samples using an optical analyzer Turbiscan AGS, previously used to evaluate the stability of vesicles and nanoparticle dispersion [23]. On the basis of TSI (Turbiscan stability index) values, we could infer that drug incorporation increases the physical stability of SLNs. In fact, as shown in - Fig. 2, both CRO- and CRT-loaded SLNs have TSI values lower than the unloaded one.

- Fig. 3 shows the DSC data of the different raw materials. Blank and drug-loaded SLNs were also prepared and characterized for comparison. Bulk Softisan 100 showed a sharp endothermic event, due to the melting, around $42^{\circ} \mathrm{C}$. When this material was formulated as SLNs, it appears broader (panel A) or in a sharp shape (panel B) and happened at a slightly higher temperature. These differences are ascribed to the different composition of the nanocarriers and to the experimental procedures employed to formulate them. The loading of CRO and CRT seems to not provoke any considerable effect in the lipid matrix thermal behavior.

- Fig. 4 shows the in vitro release profile of CRO and CRT from SLNs. The release profiles of the two drugs from the nanoparticles show a similar trend and follows the well-known "two-step drug release" related to distribution of active compounds in the SLN matrix [24]. At first, the surface bounded drug and the drug that is released immediately because of a large diffusion in concentration gradient represents the burst release. The second phase and the subsequent prolonged release could be due to the slow release of the drug from the lipid core.

As previously reported, the release profiles of the two drugs are very similar, such as the amount of drug released from the vehicles, about $60 \%$ of the loaded drug for CRT and $68 \%$ for CRO. These small differences are probably due to the different chemical characteristics of the two drugs and to the different procedures used to formulate CRO-SLNs and CRT-SLNs.

In this work, we evaluated the antioxidant effect of CRO and CRT alone and once loaded in SLNs. - Fig. 5 shows the results of the ORAC assay that we used as an analytical method to deter- 


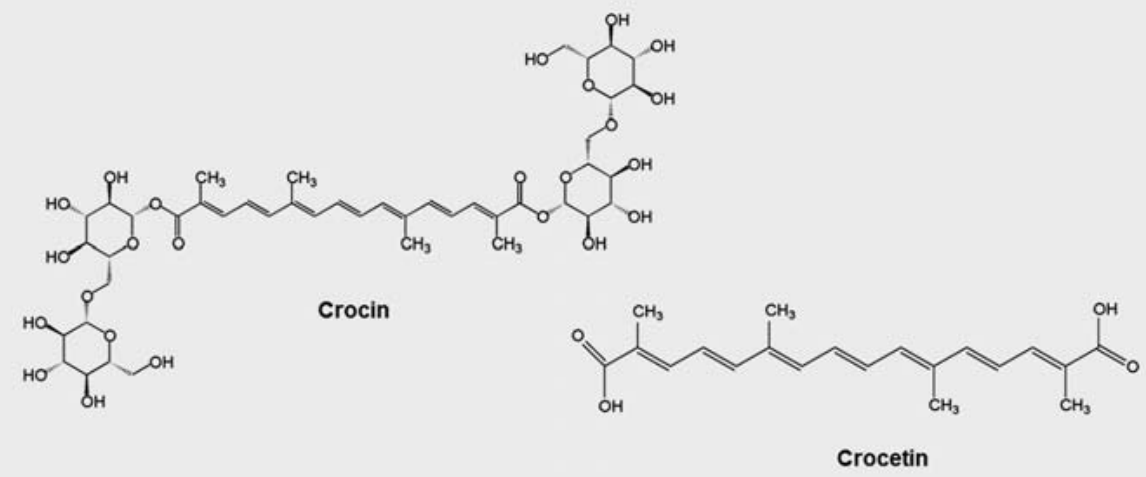

Fig. 1 Chemical structures of crocin and crocetin.

- Table 1 Particle size, polydispersivity index, and zeta potential values of different SLN-based formulations.

\begin{tabular}{|l|l|l|l|l|}
\hline Sample & $\begin{array}{l}\text { Particle size } \\
\text { (nm, mean } \pm \text { SD) }\end{array}$ & $\begin{array}{l}\text { Polidispersivity index } \\
\text { (mean } \pm \text { SD) }\end{array}$ & $\begin{array}{l}\text { Zeta potential } \\
\text { (-mV, mean } \pm \text { SD) }\end{array}$ & $\begin{array}{l}\text { Drug recovery } \\
\text { (DR \%) }\end{array}$ \\
\hline Unloaded CRO-SLNs & $248.6 \pm 19.80$ & $0.289 \pm 0.02$ & $19.3 \pm 1.54$ & - \\
\hline CRO-SLNs & $273.3 \pm 21.80$ & $0.158 \pm 0.01$ & $30.2 \pm 2.41$ & 82.1 \\
\hline Unloaded CRT-SLNs & $231.8 \pm 18.54$ & $0.258 \pm 0.02$ & $40.0 \pm 3.20$ & - \\
\hline CRT-SLNs & $278.1 \pm 22.20$ & $0.305 \pm 0.02$ & $17.8 \pm 1.42$ & 94 \\
\hline
\end{tabular}

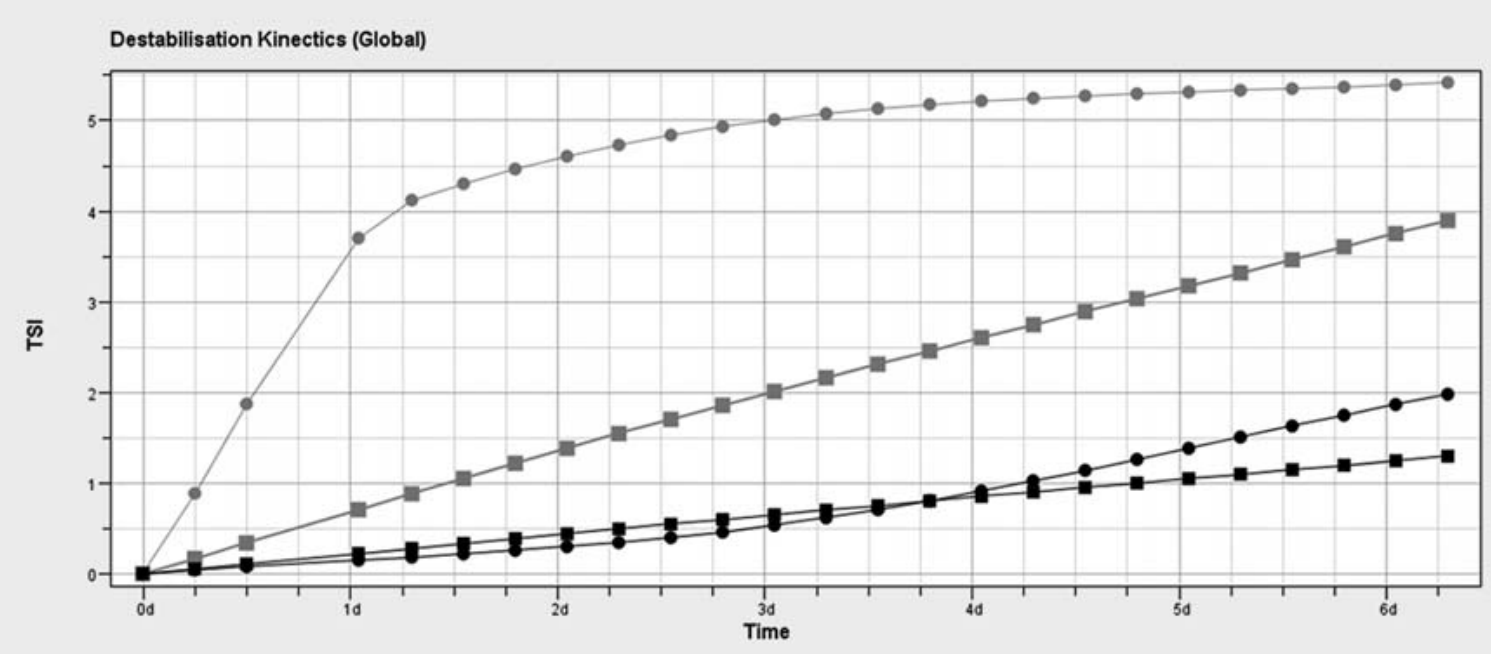

- Fig. 2 TSI values of unloaded ( $\square$ ) and crocin-loaded SLNs $(\square)$ and unloaded $(\bullet)$ and crocetin-loaded SLNs $(\bullet)$ stored at $25^{\circ} \mathrm{C}$.

mine the antioxidant potential of these carotenoids and the effect of SLNs in modulating their activity. The first evidence we obtained from the results of the ORAC assay is that CRT showed an antioxidant efficacy higher than CRO. We observed these results although CRO and CRT belong to the same family and show a similar structure. We hypothesize that the presence of the gentobiose sugar in the molecular structure of CRO modifies the conjugation degree of the active compound and consequently could influence its antioxidant activity ( $\bullet$ Fig. 5). Once encapsulated in SLNs, both CRO and CRT are able to preserve high fluorescence compared to empty SLNs or the positive control, demonstrating that the antioxidant activity of these active compounds is maintained for a longer time when it is associated to SLNs. It is noteworthy that CRT guaranteed a higher antioxidant activity compared to CRO, 


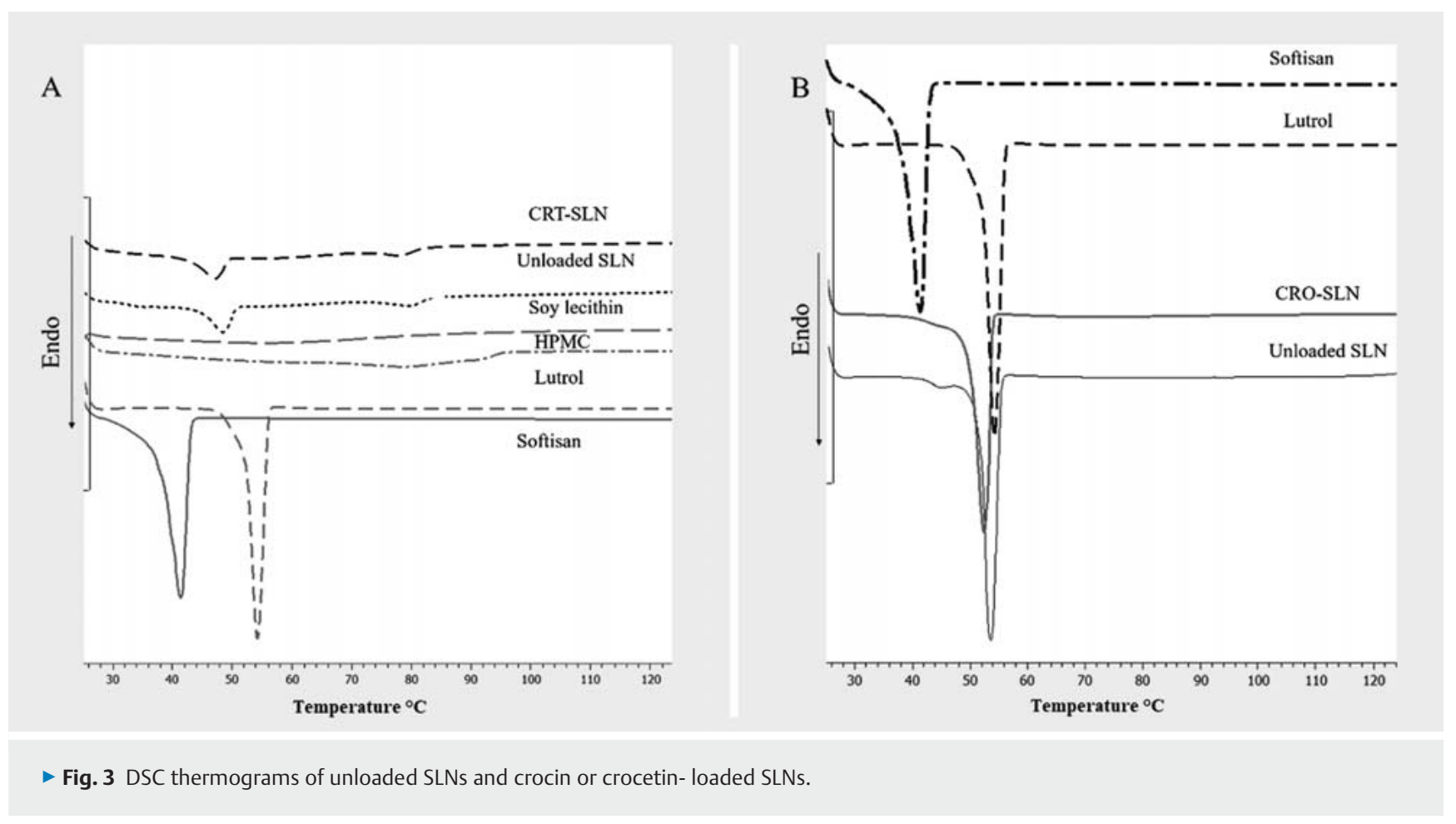

also when encapsulated in SLNs. This evidence demonstrated that SLNs are able to control the release of these active compounds, but they did not interfere in modifying their intrinsic activities.

The in vitro cytotoxic effect of CRO and CRT was evaluated by the MTT assay. We choose the cancer cell lines A375 (human melanoma) and sNF96.2 (malignant Schwann) since their use was supported by scientific evidences that demonstrated the important role of CRO and CRT in preventing and/or ameliorating some skin tumors [25] and soft tissue sarcomas in in vivo models [26]. The two cancer cells, A375 and SNF96.2, were treated with blank and drug-loaded formulations, and incubated for $72 \mathrm{~h}$. From the analysis of the data, the blank carriers did not have any important effect on cell viability, indicating the nontoxic nature of both SLNbased carriers (SLN1 and SLN2). Free drugs and SLN-based formulations showed a different antiproliferative effect against A375 and SNF96.2 cell lines ( $\triangleright$ Figs. 6 and 7). In particular, free CRO failed to induce an important cytotoxic effect with respect to control formulation for both cell lines, while CRT exhibited an extremely significant toxic effect in the same experimental conditions ( $\triangleright$ Figs. 6 and 7 ).

The nanoencapsulation strategy significantly ameliorated the cytotoxic activity of CRO for both cell lines ( $p<0.05$; $>$ Figs. 6 and 7), while CRT-SLNs did not produce a significant antiproliferative activity with respect to free CRT ( $p>0.05$; > Figs. 6 and 7).

The different trend observed between the two compounds is clearly referable to a higher chemical stability of CRT compared to CRO, while the similar cytotoxicity of free CRT and CRT-SLNs cannot exclusively be a matter of free CRT or CRT released from SLNs in the adjacent milieu, but it also depends upon selective uptake and entry of nanoparticles/bioactives inside the cells and enhanced effectiveness of bioactives released in cellular proximity.

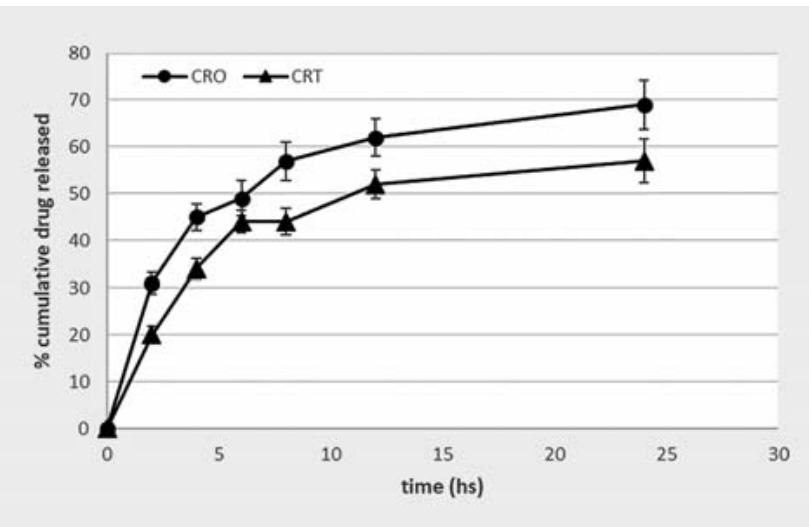

- Fig. 4 In vitro release profiles of crocin and crocetin from CROSLNs and CRT-SLNs, respectively. Each point represents the mean value $\pm S D(n=3)$.

CRT-SLNs, while ensuring an interesting cytotoxic activity in both the evaluated cell models, are not able to produce an increase in the intrinsic activity of CRT, probably because they are not able to be internalized in a satisfactory way. Therefore, we hypothesize that the observed cytotoxic effect of CRT-SLNs would depend on the release of CRT in the milieu and on the ability of the SLN system to protect the encapsulated CRT from the chemical degradation to which the compound is subjected. Besides, free CRT would have been able to produce in both models a more pronounced cytotoxic effect if it had greater chemical stability. Further studies have been planned to confirm the results obtained in the present work. 


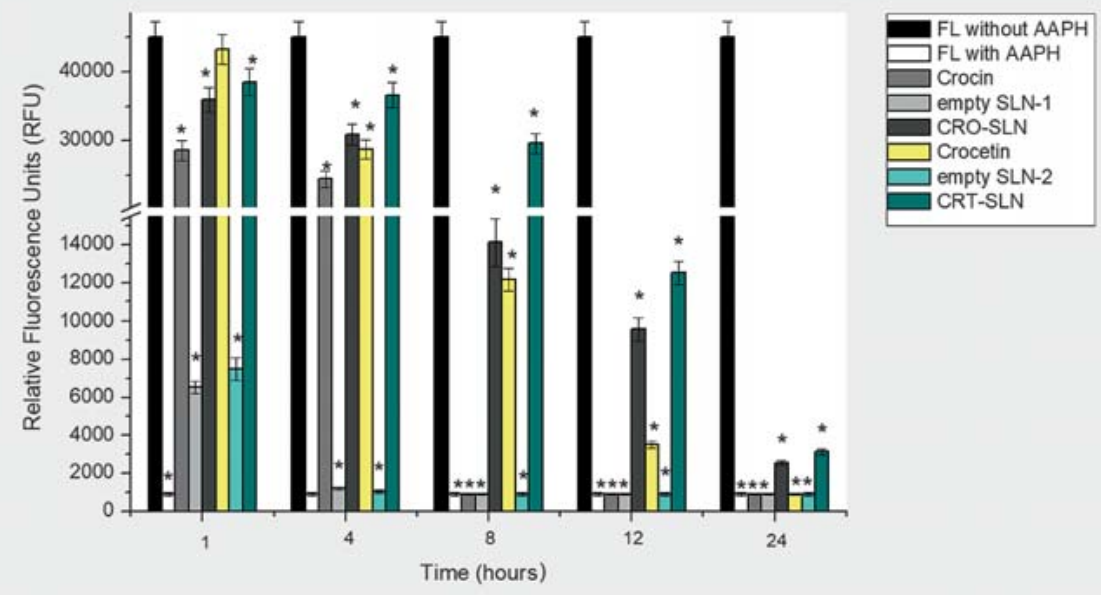

- Fig. 5 ORAC assays for CRO-SLN and CRT-SLN. Empty SLN-1 and empty SLN-2 represent the blank of CRO-SLN and CRT-SLN, respectively. Data represent the mean of three independent experiments \pm SD; ' $p<0.05$. FL: Fluorescein; AAPH: 2,2'-Azobis(2-amidinopropane) dihydrochloride; SLN: solid lipid nanoparticles; CRO: crocin; CRT: crocetin.

\section{Materials and Methods}

\section{Materials}

CRO (batch number: BCBT4979) and HPMC were purchased from Sigma-Aldrich. CRO (purity $\geq 90 \%$ ) was obtained by CRO hydrolysis following a method reported elsewhere [27]. Softisan 100 (hydrogenated coco-glycerides) was obtained by Sasol Germany. Pluronic F68 was provided from BASF. Ethanol and water were purchased from Sigma-Aldrich.

\section{Formulation of crocin-solid lipid nanoparticles and crocetin-solid lipid nanoparticles}

CRO-SLNs were prepared by using a variation of the QESD method $[22,28]$. Briefly, CRO $(0.1 \mathrm{~g})$ was solved in $5 \mathrm{~mL}$ of water heated to $50{ }^{\circ} \mathrm{C}$. The solution obtained was kept under constant magnetic stirring. The oil phase constituted by Softisan $100(0.816 \mathrm{~g})$, ethanol $(18 \mathrm{~mL})$, and water $(4 \mathrm{~mL})$ was warmed up to $50^{\circ} \mathrm{C}$ and slowly added to CRO aqueous solution. The resulting formulation was dispersed using a high-speed homogenizer (Ultra-Turrax T25, IKA-Werke $\mathrm{GmbH} \& \mathrm{Co})$ then added slowly into a hot $\left(50^{\circ} \mathrm{C}\right)$ surfactant solution ( $9 \mathrm{~mL}$ of water in which $0.7 \mathrm{~g}$ of Pluronic F68 were dissolved).

Finally, SLNs were precipitated in an ice bath by quickly adding cold water $(200 \mathrm{~mL})$ to the quasi-emulsion under high-speed homogenization. After a stirring of $30 \mathrm{~min}$, the suspension was filtered and stored at $4{ }^{\circ} \mathrm{C}$ for further characterization [28].

CRT-SLNs were prepared following the solvent diffusion method [21]. Blank and CRT-SLNs were obtained by adding CRO $(0.064 \mathrm{~g})$ to Softisan $100(0.023 \mathrm{~g})$ previously dissolved in $4.6 \mathrm{~mL}$ of ethanol at $50^{\circ} \mathrm{C}$. The aqueous phase constituted by HPMC $(0.23 \mathrm{~g})$, Pluronic F68 $(0.23 \mathrm{~g})$, soy lecithin $(0.23 \mathrm{~g})$, and water $(23 \mathrm{~mL})$ was heated to $50^{\circ} \mathrm{C}$. Then, the hot lipid phase was added to the surfactant solution and dispersed at $50^{\circ} \mathrm{C}$ for 8 min using a high-speed stirrer (Ultra Turrax T25, IKA-Werke GmbH \& Co.). The obtained pre-emulsion was ultrasonified for 10 min by using a UP $400 \mathrm{~S}$ instrument (ultrasonic processor; Dr. Hielscher $\mathrm{GmbH}$ ) and finally precipitated in an ice bath by quickly adding cold water $(100.6 \mathrm{~mL})$. The obtained SLN dispersion was filtered and later stored at $4{ }^{\circ} \mathrm{C}$.

\section{Characterization of solid lipid nanoparticle-based formulations}

Mean particle size of the lipid dispersions was measured by PCS. A Zetamaster (Malvern Instrument Ltd.), equipped with a solid-state laser having a nominal power of $4.5 \mathrm{~mW}$ with a maximum output of $5 \mathrm{~mW} 670 \mathrm{~nm}$, was employed. Analyses were performed using a $90^{\circ}$ scattering angle at $20 \pm 0.2^{\circ} \mathrm{C}$. Samples were prepared by diluting $100 \mu \mathrm{L}$ of SLN suspension, previously filtered through a 0.45- $\mu$ m Acrodisc LC 13 PVDF filter (Pall-Gelman Laboratory), with $900 \mu \mathrm{L}$ of deionized water. During the experiment, the refractive index of the samples always matched the liquid (toluene) to avoid stray light. The zeta $(\xi)$ potential was automatically calculated from electrophoretic mobility based on the Smoluchowski equation (Equation 1):

$\mathrm{v}=\left(\frac{\varepsilon \cdot \mathrm{E}}{\eta}\right) \xi$

where $v$ is the measured electrophoretic velocity, $\eta$ is the viscosity, $\varepsilon$ is the electrical permittivity of the electrolytic solution, and $E$ is the electric field. The accuracy was $0.12 \mu \mathrm{m} \mathrm{cm} / \mathrm{V} \mathrm{s}$ for aqueous systems. Samples were suspended in distilled water and the measurements were recorded at $25^{\circ} \mathrm{C}$.

\section{Determination of drug loading}

The percentage of CRO and CRT entrapped in the lipid matrix was determined as follows: an amount of CRO-SLN and CRT-SLN dispersions were filtered using a Pellicon XL tangential ultrafiltration system (Millipore) equipped with a polyethersulfone Biomax 1000 


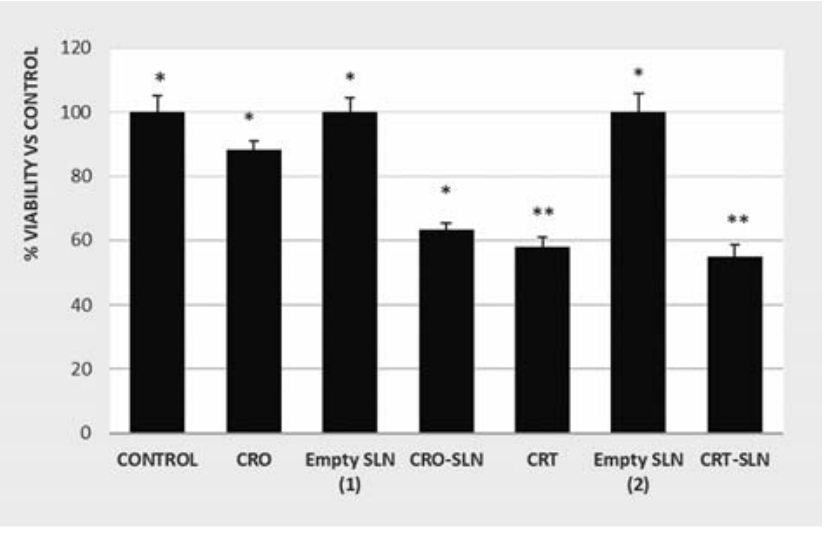

- Fig. 6 Antiproliferative activity of CRO-SLN and CRT-SLN against a human melanoma cell line (A375) versus CRO and CRT solution in PBS $(0.1 \% \mathrm{w} / \mathrm{w})$. Empty SLN-1 and empty SLN-2 represent the blank of CRO-SLN and CRT-SLN, respectively. Data represent the mean of three independent experiments $\pm S D ;{ }^{*} p<0.05 ;{ }^{* *} p>0.05$. SLN: solid lipid nanoparticle; CRO: crocin; CRT: crocetin. membrane (Millipore) with a 1000000 Da molecular weight cutoff. An amount of retained material was freeze-dried, dissolved in dichloromethane, and analyzed by UV spectrophotometry at $440 \mathrm{~nm}$ (Lambda 52, PerkinElmer) [29]. Calibration curves for the validated UV assays of CRO and CRT were performed on six solutions in the concentration range of $10-100 \mathrm{mg} / \mathrm{mL}$. The correlation coefficient was 0.99 . The incorporation efficiency was determined as DR, that is (Equation 2):

drug recovery $(\mathrm{DR} \%)=\frac{\text { mass of drug in nanoparticles }}{\text { mass of drug feed to the system }} \times 100$

\section{Differential scanning calorimetry}

Calorimetric analysis was affected by using a Mettler Toledo DSC 1 STARe system equipped with a PolyScience temperature controller. The detection system was an HSS8 high-sensitivity sensor (120 gold-gold/palladium-palladium thermocouples) and a ceramic sensor (Mettler Full Range; FRS5) with 56 thermocouples. The signal time constant was $18 \mathrm{~s}$ and the digital resolution of the measurement signal was less than $0.04 \mu \mathrm{W}$. Resolution and sensitivity calorimetric determined by TAWN test was 0.12 and 11.9 , respectively. The sampling rate was 50 values/second. The sensitivity was automatically chosen as the maximum possible by the calorimetric system, and the reference was an empty pan. The calorimetric system was calibrated, in temperature and enthalpy changes, by using indium and following the procedure of the DSC 1 Mettler TA STARe instrument. Empty and CRO- or CRTloaded SLNs were sealed in an aluminum pan and submitted to DSC analysis to determinate the influence of the drug on the thermotropic parameters of the SLNs. Each sample was submitted to heating and cooling cycles in the temperature range of $10-$ $120^{\circ} \mathrm{C}$ at a scanning rate of $5^{\circ} \mathrm{C} / \mathrm{min}$ (heating) and a scanning rate of $10^{\circ} \mathrm{C} / \mathrm{min}$ (cooling). Only free drugs were submitted to heating and cooling cycles in the temperature range of $10-200^{\circ} \mathrm{C}$ at the

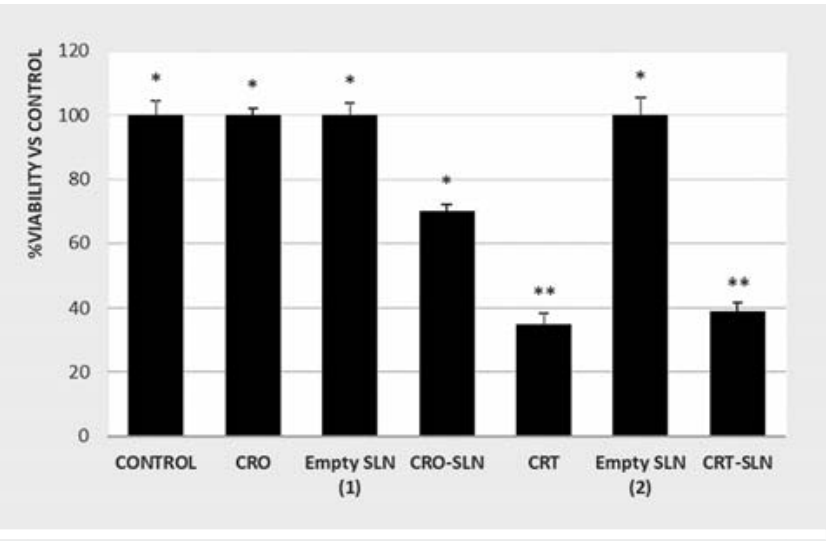

- Fig. 7 Antiproliferative activity of CRO-SLN and CRT-SLN against a malignant a Schwann cell line (sNF96.2) versus CRO and CRT solution in PBS $(0.1 \% \mathrm{w} / \mathrm{w})$. Empty SLN-1 and empty SLN-2 represent the blank of CRO-SLN and CRT-SLN, respectively. Data represent the mean of three independent experiments \pm SD; ${ }^{*} p<0.05$; ${ }^{* *} p>0.05$. SLN: solid lipid nanoparticle; CRO: crocin; CRT: crocetin.

same scanning rate. Transition temperature and enthalpy changes $(\Delta \mathrm{H})$ were calculated from peak areas with the Mettler STARe Evaluation software system (version 13.00) installed on an Optiplex 3020 DELL.

\section{Turbiscan analysis}

Turbiscan AGS (Formulaction, I'Union, France, a robot and storage station integrated TurbiscanLAB) was used to examine the dispersion stability of the SLNs. This instrument allows for the use of a simple technique to observe reversible and irreversible destabilization phenomena in the sample without the need of dilution. Turbiscan AGS is useful to detect destabilization phenomena much earlier and also in a simpler way than other methods. The suspension $(10 \mathrm{~mL})$ was placed in a flat-bottomed cylindrical glass tube and was placed in the instrument. The transmission of light from the suspensions was then measured periodically $(1 \mathrm{~h})$ along the height at 25 and $37^{\circ} \mathrm{C}$. The experiments were performed until $48 \mathrm{~h}$. The sedimentation behavior of the suspensions was monitored by measuring the backscattering and transmission of monochromatic near infrared $(\lambda=850 / 880 \mathrm{~nm})$.

\section{In vitro release study}

The amount of CRO and CRT released from SLNs was determined as reported elsewhere $[1,30]$. Briefly, a weighted amount of CRT-SLN or CRO-SLN suspension was dispersed in a phosphate buffer solution and then divided into different aliquots in glass vials. The vials were placed in a water bath that maintained a constant temperature of $37^{\circ} \mathrm{C}$. At predetermined intervals, the batch was centrifuged at $3000 \mathrm{rpm}$ for $10 \mathrm{~min}$ to separate the released drug from the SLN dispersion. After centrifugation, the sample was recovered and analyzed by a UV spectrophotometer at $440 \mathrm{~nm}$ to determine the CRT or CRO content. 


\section{Oxygen radical absorbance assay}

An ORAC assay was used to qualitatively determine the antioxidant activity of CRO-SLNs and CRT-SLNs [8]. A fresh solution of CRO and CRT alone $(25 \mu \mathrm{L})$ plus empty and loaded vehicles $(25 \mu \mathrm{L})$ were placed in 96-well tissue culture plates. One hundred and fifty $\mu \mathrm{L}$ of $\mathrm{FL}(10 \mathrm{nM})$ was used as the probe to assess the antioxidant activity. Twenty-five $\mu \mathrm{L}$ of water-soluble azo-compound AAPH $(100 \mathrm{mM})$ was used as a radical initiator to generate free radicals at a constant rate. A positive ( $F$ solution containing $\mathrm{AAPH}$ ) and negative control (FL solution containing no AAPH) were run simultaneously in PBS. A timer was started upon introduction of the free radical generator and the plate was stored in the dark at $37^{\circ} \mathrm{C}$. At each specified time point, the fluorescence of the solution was measured (excitation $492 \mathrm{~nm}$, emission $535 \mathrm{~nm}$ ) using a Wallac 1420 Victor 3 96-well plate reader fluorimeter (Perkin Elmer) and plotted as a function of time with Origin 7 software (Origin Lab Corporation).

\section{In vitro cell studies}

\section{Cell Culture}

The human melanoma A375 and malignant Schwann sNF96.2 cell lines were provided by American Type Culture Collection and routinely maintained in DMEM (Sigma-Aldrich) supplemented with $10 \%$ FBS (Invitrogen), $4 \mathrm{mM} / \mathrm{L}$ glutamine, $4500 \mathrm{mg} / \mathrm{L}$ glucose, $1500 \mathrm{mg} / \mathrm{L}$ sodium bicarbonate, $100 \mathrm{U} / \mathrm{mL}$ penicillin, and $100 \mu \mathrm{g} /$ $\mathrm{mL}$ streptomycin. Cells were incubated at $37^{\circ} \mathrm{C}$ in a humidified atmosphere of $95 \%$ air $/ 5 \% \mathrm{CO}_{2}$. A subcultivation ratio of $1: 3$ to $1: 4$ was performed twice weekly by using trypsin (0.25\%)-EDTA $(1 \mathrm{~mm})$ for the detachment. The medium was changed every 23 days.

\section{Cell treatments}

A day before the experiments, cells were trypsinized, counted, and seeded at an initial density of $8 \times 10^{3}$ cells per microwell in flat-bottomed 96 -well culture microplates (Thermo Scientific Nunc) and incubated at $37{ }^{\circ} \mathrm{C}$ in a humidified atmosphere containing $\mathrm{CO}_{2}(5 \%)$ with treatment medium (without FBS) for $24 \mathrm{~h}$. After that, the medium was removed and replaced with the treatment medium containing CRO-SLNs, CRT-SLNs, a CRO solution, a CRT solution, and a sample of empty SLNs. After $72 \mathrm{~h}$ incubation, the cells proliferation was evaluated.

\section{Cell viability evaluation}

Cell proliferation was tested by the MTT assay based on the conversion by mitochondrial dehydrogenases of a substrate containing a tetrazolium ring into blue formazan, detectable spectrophotometrically. At different time points, the treatment media were removed and a solution of MTT $(0.5 \mathrm{mg} / \mathrm{mL})$ was added to each microwell. After $3 \mathrm{~h}$ of incubation at $37^{\circ} \mathrm{C}$, the supernatant was removed and replaced with DMSO $(100 \mu \mathrm{L})$. The optical density of each well sample was measured with a microplate spectrophotometer reader (Digital and Analog Systems) at $\lambda=550 \mathrm{~nm}$. Each sample was tested in quadruplicate $(n=12)$ and cell viability was reported as percentage (\%) compared to the controls (untreated, empty SLNs).

\section{Statistical analysis}

Statistical analysis of the data was performed using Student's ttest. A probability (p) of less than 0.05 was considered significant in this study. All statistical analyses were performed using the statistical software package SYSTAT, version 13 (Systat Inc.).

Conflict of Interest

The authors declare no conflict of interest

References

[1] Rahaiee S, Hashemi M, Shojaosadati SA, Moini S, Razavi SH. Nanoparticles based on crocin loaded chitosan-alginate biopolymers: Antioxidant activities, bioavailability and anticancer properties. Int J Biol Macromol 2017; 99: 401-408

[2] Rahaiee S, Moini S, Hashemi M, Shojaosadati SA. Evaluation of antioxidant activities of bioactive compounds and various extracts obtained from saffron (Crocus sativus L.): a review. J Food Sci Technol 2015; 52: $1881-1888$

[3] Abdullaev Fl. Biological effects of saffron. Biofactors 1993; 4: 83-86

[4] Gutheil WG, Reed G, Ray A, Anant S, Dhar A. Crocetin: an agent derived from saffron for prevention and therapy for cancer. Curr Pharm Biotechnol 2012; 13: 173-179

[5] Nam KN, Park YM, Jung HJ, Lee JY, Min BD, Park SU, Jung WS, Cho KH, Park JH, Kang I, Hong JW, Lee EH. Anti-inflammatory effects of crocin and crocetin in rat brain microglial cells. Eur J Pharmacol 2010; 648: $110-116$

[6] Yaribeygi H, Mohammadi MT, Sahebkar A. Crocin potentiates antioxidant defense system and improves oxidative damage in liver tissue in diabetic rats. Biomed Pharmacother 2018; 98: 333-337

[7] Finley JW, Gao S. A perspective on Crocus sativus L. (saffron) constituent crocin: a potent water-soluble antioxidant and potential therapy for Alzheimer's disease. J Agric Food Chem 2017; 65: 1005-1020

[8] Esposito E, Drechsler M, Mariani P, Panico AM, Cardile V, Crascì L, Carducci F, Graziano ACE, Cortesi R, Puglia C. Nanostructured lipid dispersions for topical administration of crocin, a potent antioxidant from saffron (Crocus sativus L.). Mater Sci Eng C Mater Biol Appl 2017; 71: 669-677

[9] Baghishani F, Mohammadipour A, Hosseinzadeh $H$, Hosseini M, Ebrahimzadeh-Bideskan A. The effects of tramadol administration on hippocampal cell apoptosis, learning and memory in adult rats and neuroprotective effects of crocin. Metab Brain Dis 2018; 33: 907-916

[10] Wang X, Yuan B, Cheng B, Liu Y, Zhang B, Wang X, Lin X, Yang B, Gong G. Crocin alleviates myocardial ischemia/reperfusion-induced endoplasmic reticulum stress via regulation of MIR-34A/SIRT1/NRF2 pathway. Shock 2018. doi: $10.1097 /$ SHK.0000000000001116

[11] Li X, Jiang C, Zhu W. Crocin reduces the inflammation response in rheumatoid arthritis. Biosci Biotechnol Biochem 2017; 81: 891-898

[12] Yao C, Liu BB, Qian XD, Li LQ, Cao HB, Guo QS, Zhou GF. Crocin induces autophagic apoptosis in hepatocellular carcinoma by inhibiting Akt/ mTOR activity. Onco Targets Ther 2018; 11: 2017-2028

[13] Kim B, Park B. Saffron carotenoids inhibit STAT3 activation and promote apoptotic progression in IL-6-stimulated liver cancer cells. Oncol Rep 2018; 39: 1883-1891

[14] Tsimidou M, Biliaderis CG. Kinetic studies of saffron (Crocus sativus L.) quality deterioration. J Agric Food Chem 1997; 45: 2890-2898

[15] Tsimidou M, Tsatsaroni E. Stability of saffron pigments in aqueous extracts. J Food Sci 1993; 58: 1073-1075 
[16] Asai A, Nakano T, Takahashi M, Nagao A. Orally administered crocetin and crocins are absorbed into blood plasma as crocetin and its glucuronide conjugates in mice. J Agric Food Chem 2005; 53: 7302-7306

[17] Kanakis CD, Tarantilis PA, Tajmir-Riahi HA, Polissiou MG. Crocetin, dimethylcrocetin, and safranal bind human serum albumin: stability and antioxidative properties. J Agric Food Chem 2007; 55: 970-977

[18] Puglia C, Rizza L, Drechsler M, Bonina F. Nanoemulsions as vehicles for topical administration of glycyrrhetic acid: characterization and in vitro and in vivo evaluation. Drug Deliv 2010; 17: 123-129

[19] Tapeinos C, Battaglini M, Ciofani G. Advances in the design of solid lipid nanoparticles and nanostructured lipid carriers for targeting brain diseases. J Control Release 2017; 264: 306-332

[20] Puglia C, Offerta A, Carbone C, Bonina F, Pignatello R, Puglisi G. Lipid nanocarriers (LNC) and their applications in ocular drug delivery. Curr Med Chem 2015; 22: 1589-1602

[21] Gallarate M, Trotta M, Battaglia L, Chirio D. Preparation of solid lipid nanoparticles from W/O/W emulsions: preliminary studies on insulin encapsulation. J Microencapsul 2009; 26: 394-402

[22] Kawashima Y, Niwa T, Handa T, Takeuchi H, Iwamoto T, Itoh Y. Preparation of prolonged-release spherical micro-matrix of ibuprofen with acrylic polymer by the emulsion-solvent diffusion method for improving bioavailability. Chem Pharm Bull 1989; 37: 425-429

[23] Carbone C, Campisi A, Manno D, Serra A, Spatuzza M, Musumeci T, Bonfanti R, Puglisi G. The critical role of didodecyldimethylammonium bromide on physico-chemical, technological and biological properties of NLC. Colloids Surf B Biointerfaces 2014; 121: 1-10

[24] Geszke-Moritz M, Moritz M. Solid lipid nanoparticles as attractive drug vehicles: Composition, properties and therapeutic strategies. Mater Sci Eng C Mater Biol Appl 2016; 68: 982-994

[25] Das I, Chakrabarty RN, Das S. Saffron can prevent chemically induced skin carcinogenesis in Swiss albino mice. Asian Pacific J Cancer Prev 2004; 5: 70-76

[26] Abdullaev F. Cancer chemopreventive and tumoricidal properties of saffron (Crocus sativus L.). Exp Biol Med 2002; 227: 20-25

[27] Takahashi M, Harada K. Method for the purification of crocetin. Japan Patent WO2004078695; 2004

[28] Puglia C, Offerta A, Tirendi GG, Tarico MS, Curreri S, Bonina F, Perrotta RE. Design of solid lipid nanoparticles for caffeine topical administration. Drug Deliv 2016; 23: 36-40

[29] Lage M, Cantrell CL. Quantification of saffron (Crocus sativus L.) metabolites crocins, picrocrocin and safranal for quality determination of the spice grown under different environmental Moroccan conditions. Sci Hortic 2009; 121: 366-373

[30] Puglia C, Frasca G, Musumeci T, Rizza L, Puglisi G, Bonina F, Chiechio S. Curcumin loaded NLC induces histone hypoacetylation in the CNS after intraperitoneal administration in mice. Eur J Pharm Biopharm 2012; 81: 288-293 